\title{
Analysis of Inflection and Singular Points on a Parametric Curve with a Shape Factor
}

\author{
Zhi Liu ${ }^{1}$, Chen $\mathrm{Li}^{2}$, Jieqing Tan ${ }^{1}$ and Xiaoyan Chen ${ }^{1, *}$ \\ 1 School of Mathematics, Hefei University of Technology, Hefei 23009, China; \\ liuzhi314@126.com (Z.L.); jieqingtan@hfut.edu.cn (J.T.) \\ 2 College of Information \& Network Engineering, Anhui Science and Technology University, \\ Chuzhou 233100, China; lichen20160920@126.com \\ * Correspondence: chenxiaoyan314@126.com
}

Academic Editor: Chungang Zhu

Received: 19 September 2016; Accepted: 11 January 2017; Published: 19 January 2017

\begin{abstract}
The features of a class of cubic curves with a shape factor are analyzed by means of the theory of envelope and topological mapping. The effects of the shape factor on the cubic curves are made clear. Necessary and sufficient conditions are derived for the curve to have one or two inflection points, a loop or a cusp, or to be locally or globally convex. Those conditions are completely characterized by the relative position of the edge vectors of the control polygon and the shape factor. The results are summarized in a shape diagram, which is useful when the cubic parametric curves are used for geometric modeling. Furthermore, we discuss the influences of the shape factor on the shape diagram and the ability for adjusting the shape of the curve.
\end{abstract}

Keywords: shape factor; singular points; inflection points; local convexity; global convexity

\section{Introduction}

Bézier curves and surfaces are modeling tools widely used in CAD/CAM (computer-aided design and computer-aided manufacturing) systems [1]. The use of Bernstein polynomials as the basis functions in Bézier's UNISURF [2] is well known. Cubic Bernstein basis functions are represented by:

$$
\left\{(1-u)^{3}, 3(1-u)^{2} u, 3 u^{2}(1-u), u^{3}\right\} .
$$

In the CONSURF system developed by Ball [3-5] at the British Aircraft Corporation, the following basis for cubic polynomials was used:

$$
\left\{(1-u)^{2}, 2(1-u)^{2} u, 2 u^{2}(1-u), u^{2}\right\} .
$$

Said [6] extended it to arbitrary odd degrees, namely the generalized Ball curves. The generalized Ball curves possess many nice properties which are similar to those of Bézier curves, such as computational stability, the symmetry property, the convex hull property, endpoint interpolation, the geometric invariant [7]. The generalized Ball representations for a polynomial curve are much better suited to degree-raising and -lowering than Bézier representations. It is well known that degree elevation and reduction are important in transferring data between various CAD systems. Goodman and Said [8,9] suggest that, in the situation where degree elevation and reduction are important, while other process are less important, the designer of curves and surfaces should consider using the generalized Ball form instead of the Bézier form.

In CAD/CAGD (computer-aided geometric design), it is often necessary to detect inflection points and singularities on curves. Convexity is an important intuitive geometric concept and convexity 
control of curves and surfaces plays a fundamental role. For planar cubic Bézier curves an exhaustive study was presented in [10] and for the rational case in [11]. Manocha and Canny [12] studied this problem for polynomial and rational parametric curves of arbitrary degree. Yang and Wang [13] discussed inflection points and singularities on C-Bézier curves, and the results are summarized in a shape diagram of C-Bézier curves. Juhász [14] detected cusps, inflection points, and loops of C-Bézier curves by letting a control point vary, while the rest are held fixed. However, local and global convexity is not referred to. There are many other publications on this topic [15].

With the in-depth study of curve representation, there have been more new curves similar to the Bézier curves and the Ball curves; for example, Q-Bézier curves [16]. Due to the introduction of the shape parameter, these curves are highlighting the flexibility to change the shape of the curve in geometric modeling. In order to have insight into the influence that the shape parameters exert on geometrical characteristics of the curve, we need to further study the conditions for the curve to have inflection points, loops or cusps, or to be locally or globally convex.

This paper is organized as follows: First we show the construction of a class of cubic parametric curves with a variable shape factor. The Ball curve, the cubic Bézier curve, and the cubic Timmer curve are special cases of the curve. In Section 3, the inflection points and singularities of the space cubic parametric curves are discussed. In Section 4, shape features of the planar cubic parametric curves are proposed by using the method based on the theory of envelopes and topological mappings. Necessary and sufficient conditions are derived for this curve to have one or two inflection points, a loop or a cusp, to be locally or globally convex. The results are summarized in a shape diagram. Finally, the influences of shape factor on the shape diagram and their ability for adjusting the shape of the curve are analyzed.

\section{The Cubic Parametric Curve with a Shape Factor}

Definition 1. Given four control points $\boldsymbol{P}_{i} \in \boldsymbol{R}^{d}(d=2,3, i=0,1,2,3)$, the cubic parametric curve with a shape factor is defined as follows:

$$
\boldsymbol{P}(t)=\sum_{i=0}^{3} \boldsymbol{P}_{i} B_{i}(t), \quad t \in[0,1]
$$

where $B_{i}(t)(i=0,1,2,3)$ are the basis functions with the shape factor $\lambda$ defined by:

$$
\begin{aligned}
& B_{0}(t)=[1+(2-\lambda) t](1-t)^{2}, \quad B_{1}(t)=\lambda t(1-t)^{2}, \\
& B_{2}(t)=\lambda t^{2}(1-t), \quad B_{3}(t)=[1+(2-\lambda)(1-t)] t^{2} .
\end{aligned}
$$

If $\lambda=0$, the cubic parametric curve degenerates into a straight line. If $\lambda=2$, the cubic parametric curve degenerates into a Ball curve. If $\lambda=3$, the cubic parametric curve degenerates into a cubic Bézier curve. If $\lambda=4$, the cubic parametric curve degenerates into a cubic Timmer curve [17]. Thus, the Ball curve, the cubic Bézier curve, and the cubic Timmer curve are all special cases of the cubic parametric curve defined in Equation (1).

When the parameter $\lambda \in(0,3]$, the cubic parametric curves have similar properties to cubic Bézier curves or Ball curves, such as symmetry, the endpoint interpolation, end edge tangent, convex hull property and geometrical invariance. The cubic parametric curve also has a similar recursive evaluation, degree elevation, and reduction algorithms. Thus, we assume that $\lambda \in(0,3]$ in this paper.

The introduction of the variable shape factor makes the curve shape feature distribution simpler and easier to control. Given four control points, we can globally or locally adjust the shape of the curve by changing the shape factor value. The cubic parametric curve is more approximate to the control polygon with the increasing shape factor $\lambda$, otherwise farther away from the control polygon. 
Therefore, the cubic parametric curve is more flexible in adjusting the shape of the curve than the cubic Bézier curve or Ball curve.

Figure 1 shows the cubic parametric curves with shape factor $\lambda=1, \lambda=2$ (Ball curve), $\lambda=3$ (cubic Bézier curve), and $\lambda=4$ (cubic Timmer curve), respectively.

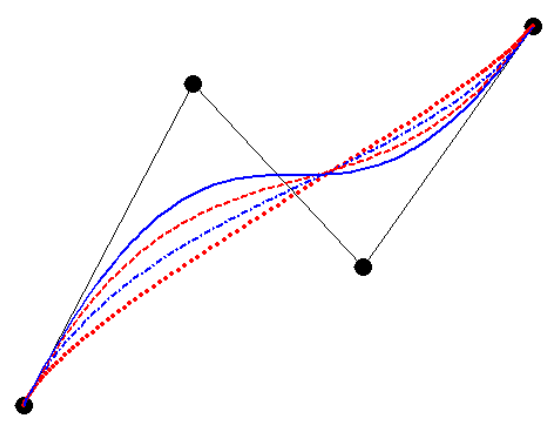

(a)

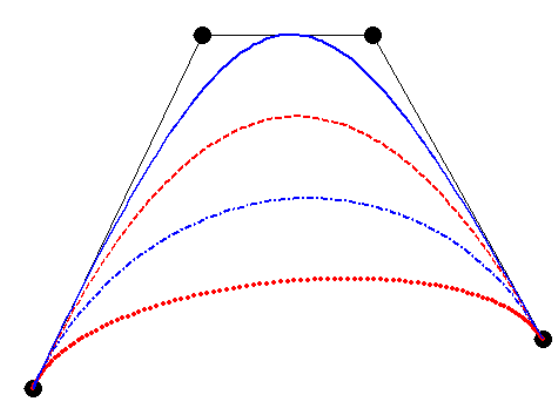

(b)

Figure 1. The cubic parametric curves with a shape factor (red dotted lines, $\lambda=1$; blue dash dotted lines, $\lambda=2$; red dashed lines, $\lambda=3$; blue solid lines, $\lambda=4$ ) (a) The cubic parametric curves with single inflection point; (b) The cubic parametric curves without inflection point.

Figure 2 shows different shapes of five-pointed star petals. Taking into account the symmetry of the petal shape, we take $\lambda_{i}=\lambda, i=1, \cdots, 5$.

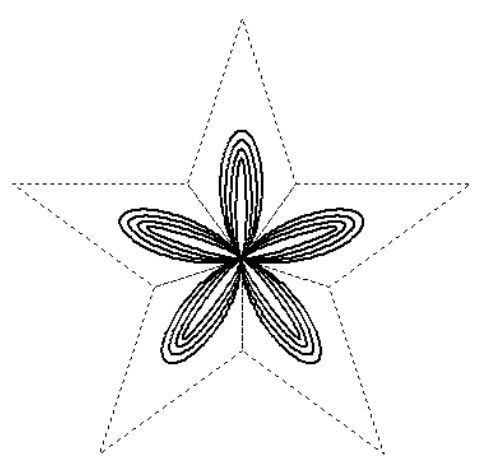

Figure 2. Five-pointed star petal modeling $(\lambda=0,1,2,3)$.

\section{Geometric Features of the Space Cubic Curve}

In practical applications, geometric features, such as the singularity, inflection point, cusp, and convex distribution of curve, are very important to determine the shape of the curve.

Theorem 1. If $\lambda \in(0,3]$ and the control points $\boldsymbol{P}_{i} \in \boldsymbol{R}^{3}(i=0,1,2,3)$ are not coplanar, then the cubic parametric curve $\boldsymbol{P}(t)$ has no singular point, cusp, double point, or pan inflection point, and the direction of rotation of the curve $\boldsymbol{P}(t)$ is consistent with that of the control polygon.

Proof. First, we prove that the curve $\boldsymbol{P}(t)$ has no cusp. Let $\boldsymbol{q}_{i}=\boldsymbol{P}_{i}-\boldsymbol{P}_{i-1}(i=1,2,3)$ be the edge vectors of the control polygon. Then $\boldsymbol{P}(t)$ can be simplified to:

$$
\boldsymbol{P}(t)=\boldsymbol{P}_{0}+\left[1-B_{0}(t)\right] \boldsymbol{q}_{1}+\left[B_{2}(t)+B_{3}(t)\right] \boldsymbol{q}_{2}+B_{3}(t) \boldsymbol{q}_{3}
$$

Therefore:

$$
\boldsymbol{P}^{\prime}(t)=-B_{0}^{\prime}(t) \boldsymbol{q}_{1}+\left[B_{2}^{\prime}(t)+B_{3}^{\prime}(t)\right] \boldsymbol{q}_{2}+B_{3}^{\prime}(t) \boldsymbol{q}_{3} .
$$


When $t \in(0,1)$, it follows from Equation (2) that:

$$
B_{2}^{\prime}(t)+B_{3}^{\prime}(t)=6 t(1-t) \neq 0
$$

Since the control points $\boldsymbol{P}_{i}(i=0,1,2,3)$ are not coplanar, the edge vectors $\boldsymbol{q}_{i}(i=1,2,3)$ are linearly independent; so $\boldsymbol{P}^{\prime}(t) \neq \mathbf{0}$. Therefore, the curve $\boldsymbol{P}(t)$ has no cusp.

Next, we prove that the curve $\boldsymbol{P}(t)$ has no double point. Assume that the curve $\boldsymbol{P}(t)$ has a double point, say, $\boldsymbol{P}\left(t_{1}\right)-\boldsymbol{P}\left(t_{2}\right)=0$, where $0 \leq t_{1}<t_{2} \leq 1$. Then it follows from Equation (3) that:

$$
\left[B_{0}\left(t_{2}\right)-B_{0}\left(t_{1}\right)\right] \boldsymbol{q}_{1}+\left[B_{2}\left(t_{1}\right)+B_{3}\left(t_{1}\right)-B_{2}\left(t_{2}\right)-B_{3}\left(t_{2}\right)\right] \boldsymbol{q}_{2}+\left[B_{3}\left(t_{1}\right)-B_{3}\left(t_{2}\right)\right] \boldsymbol{q}_{3}=0
$$

Since the edge vectors $\boldsymbol{q}_{i}(i=1,2,3)$ are linearly independent, we have $B_{i}\left(t_{1}\right)=B_{i}\left(t_{2}\right), i=0,2,3$. The equation $B_{0}\left(t_{1}\right)=B_{0}\left(t_{2}\right)$ implies that there exists $\xi \in\left(t_{1}, t_{2}\right) \subseteq[0,1]$ such that $B_{0}^{\prime}(\xi)=0$, namely, $\xi=\frac{\lambda}{3(\lambda-2)}$. Since $0<\xi<1$, we have $0<\frac{\lambda}{3(\lambda-2)}<1$, which results in $\lambda>3$ or $\lambda<0$, contradicting $\lambda \in(0,3]$. Therefore, $B_{0}\left(t_{1}\right) \neq B_{0}\left(t_{2}\right)$. Hence the curve $\boldsymbol{P}(t)$ has no double point.

Finally, we prove that the curve $\boldsymbol{P}(t)$ has no pan inflection points, and the direction of rotation of $\boldsymbol{P}(t)$ is consistent with that of the polygon.

The point $\boldsymbol{P}\left(t_{0}\right)\left(0<t_{0}<1\right)$ is the pan inflection point of the space curve $\boldsymbol{P}(t)$ if and only if the sign of torsion changes when it passes through $t_{0}$. We assume:

$$
g(t)=\operatorname{det}\left(\boldsymbol{P}^{\prime}(t), \boldsymbol{P}^{\prime \prime}(t), \boldsymbol{P}^{\prime \prime \prime}(t)\right)
$$

Note that:

$$
\sum_{i=0}^{3} B_{i}(t)=1, \sum_{i=0}^{3} B_{i}^{\prime}(t)=\sum_{i=0}^{3} B_{i}^{\prime \prime}(t)=\sum_{i=0}^{3} B_{i}^{\prime \prime \prime}(t)=0
$$

Then:

$$
\begin{gathered}
g(t)=\operatorname{det}\left[\sum_{i=0}^{3} \boldsymbol{P}_{i} B_{i}^{\prime}(t) \sum_{i=0}^{3} \boldsymbol{P}_{i} B_{i}^{\prime \prime}(t) \sum_{i=0}^{3} \boldsymbol{P}_{i} B_{i}^{\prime \prime \prime}(t)\right]=\left|\begin{array}{llll}
\sum_{i=0}^{3} B_{i}(t) & \sum_{i=0}^{3} B_{i}^{\prime}(t) & \sum_{i=0}^{3} B_{i}^{\prime \prime}(t) & \sum_{i=0}^{3} B_{i}^{\prime \prime \prime}(t) \\
\sum_{i=0}^{3} \boldsymbol{P}_{i} B_{i}(t) & \sum_{i=0}^{3} \boldsymbol{P}_{i} B_{i}^{\prime}(t) & \sum_{i=0}^{3} \boldsymbol{P}_{i} B_{i}^{\prime \prime}(t) & \sum_{i=0}^{3} \boldsymbol{P}_{i} B_{i}^{\prime \prime \prime}(t)
\end{array}\right| \\
\left.=\mid \begin{array}{ccc|ccc}
1 & 1 & 1 & 1 \\
\boldsymbol{P}_{0} & \boldsymbol{P}_{1} & \boldsymbol{P}_{2} & \boldsymbol{P}_{3}
\end{array}\right]\left[\begin{array}{cccc}
B_{0}(t) & B_{0}^{\prime}(t) & B_{0}^{\prime \prime}(t) & B_{0}^{\prime \prime \prime}(t) \\
B_{1}(t) & B_{1}^{\prime}(t) & B_{1}^{\prime \prime}(t) & B_{1}^{\prime \prime \prime}(t) \\
B_{2}(t) & B_{2}^{\prime}(t) & B_{2}^{\prime \prime}(t) & B_{2}^{\prime \prime \prime}(t) \\
B_{3}(t) & B_{3}^{\prime}(t) & B_{3}^{\prime \prime}(t) & B_{3}^{\prime \prime \prime}(t)
\end{array}\right] \mid \\
=\left|\begin{array}{cccccc}
B_{0}(t) & B_{0}^{\prime}(t) & B_{0}^{\prime \prime}(t) & B_{0}^{\prime \prime \prime}(t) & \\
B_{1}(t) & B_{1}^{\prime}(t) & B_{1}^{\prime \prime}(t) & B_{1}^{\prime \prime \prime}(t) \\
B_{2}(t) & B_{2}^{\prime}(t) & B_{2}^{\prime \prime}(t) & B_{2}^{\prime \prime \prime}(t) \\
B_{3}(t) & B_{3}^{\prime}(t) & B_{3}^{\prime \prime}(t) & B_{3}^{\prime \prime \prime}(t)
\end{array}\right|=\left(\boldsymbol{q}_{1}, \boldsymbol{q}_{2}, \boldsymbol{q}_{3}\right) D(t),
\end{gathered}
$$

where $\left(q_{1}, q_{2}, q_{3}\right)$ is the mixed product of the edge vectors $q_{1}, q_{2}, q_{3}$. The edge vectors $q_{1}, q_{2}, q_{3}$ are not coplanar, so $\left(\boldsymbol{q}_{1}, \boldsymbol{q}_{2}, \boldsymbol{q}_{3}\right) \neq 0$. Since $\lambda \in(0,3]$, we get:

$$
D(t)=\left|\begin{array}{llll}
B_{0}(t) & B_{0}^{\prime}(t) & B_{0}^{\prime \prime}(t) & B_{0}^{\prime \prime \prime}(t) \\
B_{1}(t) & B_{1}^{\prime}(t) & B_{1}^{\prime \prime}(t) & B_{1}^{\prime \prime \prime}(t) \\
B_{2}(t) & B_{2}^{\prime}(t) & B_{2}^{\prime \prime}(t) & B_{2}^{\prime \prime \prime}(t) \\
B_{3}(t) & B_{3}^{\prime}(t) & B_{3}^{\prime \prime}(t) & B_{3}^{\prime \prime \prime}(t)
\end{array}\right|=12 \lambda^{2}>0 .
$$


For $0 \leq t \leq 1$, we have $g(t) \neq 0$ and $g(t)$ has the same sign as $\left(\boldsymbol{q}_{1}, \boldsymbol{q}_{2}, \boldsymbol{q}_{3}\right)$. Thus, the cubic parametric curve $\boldsymbol{p}(t)$ has no pan inflection point and the direction of rotation of the curve $p(t)$ is consistent with that of the polygon. The proof of Theorem 1 is completed.

\section{Geometric Features of the Planar Cubic Curve}

It is known that a planar cubic parametric curve may have one or two inflection points, a loop or a cusp. If the control points $\boldsymbol{P}_{i} \in \boldsymbol{R}^{3}(i=0,1,2,3)$ are coplanar, then edge vectors $\boldsymbol{q}_{i}(i=1,2,3)$ are linearly dependent and the cubic parametric curve $p(t)$ reduces to a plane curve. The following discussion is based on the positional relationship of $\boldsymbol{q}_{1}$ and $\boldsymbol{q}_{3}$.

\subsection{Edge Vectors $\boldsymbol{q}_{1}$ and $\boldsymbol{q}_{3}$ Are Non-Parallel}

When edge vectors $\boldsymbol{q}_{1}$ and $\boldsymbol{q}_{3}$ are non-parallel, $\boldsymbol{q}_{1}$ and $\boldsymbol{q}_{3}$ are the base vectors of the plane. Let $\boldsymbol{q}_{2}=u \boldsymbol{q}_{1}+v \boldsymbol{q}_{3}$. From Equation (3), we have:

$$
\begin{aligned}
\boldsymbol{p}(t)=\boldsymbol{P}_{0} & +\left[1-B_{0}(t)+u\left(B_{2}(t)+B_{3}(t)\right)\right] \boldsymbol{q}_{1} \\
& +\left[B_{3}(t)+v\left(B_{2}(t)+B_{3}(t)\right)\right] \boldsymbol{q}_{3} .
\end{aligned}
$$

If $p^{\prime}(t)=\mathbf{0}(0<t<1)$, then:

$$
\left[-B_{0}^{\prime}(t)+u\left(B_{2}^{\prime}(t)+B_{3}^{\prime}(t)\right)\right] \boldsymbol{q}_{1}+\left[B_{3}^{\prime}(t)+v\left(B_{2}^{\prime}(t)+B_{3}^{\prime}(t)\right)\right] \boldsymbol{q}_{3}=\mathbf{0} .
$$

Since $\boldsymbol{q}_{1}$ and $\boldsymbol{q}_{3}$ are linearly independent, we have:

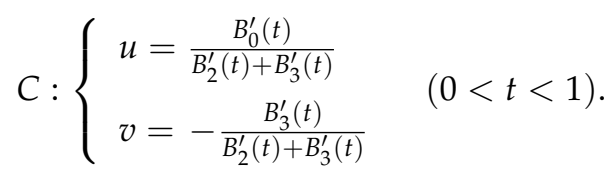

Substituting Equation (2) into the above two equations gives:

$$
C:\left\{\begin{array}{l}
u=\frac{\lambda}{2}-1-\frac{\lambda}{6 t} \\
v=\frac{\lambda}{2}-1-\frac{\lambda}{6(1-t)}
\end{array} \quad(0<t<1) .\right.
$$

Next, we discuss the shape of the curve C. From Equation (6), we know:

$$
\lim _{t \rightarrow 0^{+}} u=-\infty, \lim _{t \rightarrow 0^{+}} v=\frac{\lambda}{3}-1, \lim _{t \rightarrow 1^{-}} u=\frac{\lambda}{3}-1, \lim _{t \rightarrow 1^{-}} v=-\infty .
$$

Thus. the curve $C$ has two asymptotes: $u=\frac{\lambda}{3}-1, v=\frac{\lambda}{3}-1$. On the other hand, we get, from Equation (6), that:

$$
\frac{\mathrm{d} v}{\mathrm{~d} u}=-\frac{t^{2}}{(t-1)^{2}}<0, \quad \frac{\mathrm{d}^{2} v}{\mathrm{~d} u^{2}}=\frac{12 t^{3}}{\lambda(t-1)^{3}}<0
$$

Therefore, the curve $C$ is monotonic decreasing $(0<t<1, \lambda \in(0,3])$ and has no inflection point.

By means of the monotone and strict convexity of the curve $C$, we further discuss the cusps, the inflection points and convexity of the curve $\boldsymbol{p}(t)$.

\subsubsection{About the Cusp}

The necessary condition for the curve $p(t)$ to have a cusp is:

$$
p^{\prime}(t)=0(0<t<1) \text {. }
$$


Suppose $t_{0}\left(0<t_{0}<1\right)$ is the point corresponding to $\left(u_{0}, v_{0}\right) \in C$, such that $p^{\prime}\left(t_{0}\right)=0$.

The Taylor expansion of $\boldsymbol{p}(t)$ about $t_{0}$ is:

$$
\boldsymbol{p}(t)=\boldsymbol{p}\left(t_{0}\right)+\boldsymbol{p}^{\prime}\left(t_{0}\right)\left(t-t_{0}\right)+\frac{1}{2} \boldsymbol{p}^{\prime \prime}\left(t_{0}\right)\left(t-t_{0}\right)^{2}+o\left(t-t_{0}\right)^{2} .
$$

Differentiating the above equation yields:

$$
p^{\prime}(t)=p^{\prime \prime}\left(t_{0}\right)\left(t-t_{0}\right)+o\left(t-t_{0}\right),
$$

where $\boldsymbol{p}^{\prime \prime}\left(t_{0}\right) \neq 0$. In fact, by Equation (5) $\boldsymbol{p}^{\prime \prime}(t)=\mathbf{0}(0<t<1)$ implies:

$$
\left[-B_{0}^{\prime \prime}(t)+u\left(B_{2}^{\prime \prime}(t)+B_{3}^{\prime \prime}(t)\right)\right] \boldsymbol{q}_{1}+\left[B_{3}^{\prime \prime}(t)+v\left(B_{2}^{\prime \prime}(t)+B_{3}^{\prime \prime}(t)\right)\right] \boldsymbol{q}_{3}=\mathbf{0} .
$$

Since $\boldsymbol{q}_{1}$ and $\boldsymbol{q}_{3}$ are linearly independent, we have:

$$
\left\{\begin{array}{l}
u=\frac{B_{0}^{\prime \prime}(t)}{B_{2}^{\prime \prime}(t)+B_{3}^{\prime \prime}(t)} \\
v=\frac{-B_{3}^{\prime \prime}(t)}{B_{2}^{\prime \prime}(t)+B_{3}^{\prime \prime}(t)}
\end{array} \quad(0<t<1)\right.
$$

That is:

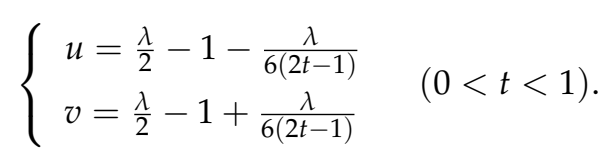

If Equations (6) and (7) hold simultaneously, then we obtain $\lambda=0$, contradicting $\lambda \in(0,3]$; so $p^{\prime \prime}\left(t_{0}\right) \neq 0$.

While $\boldsymbol{p}^{\prime}\left(t_{0}\right)=0, \boldsymbol{p}^{\prime \prime}\left(t_{0}\right) \neq 0$, we know the direction of $\boldsymbol{p}^{\prime}(t)$ changes when it passes through $t_{0}$. As a result, $\boldsymbol{p}\left(t_{0}\right)$ is a cusp on the curve $\boldsymbol{p}(t)$. Therefore, the curve $\boldsymbol{p}(t)$ having a cusp is equivalent to $(u, v) \in C$.

\subsubsection{About the Inflection Point}

The point $p\left(t_{0}\right)\left(0<t_{0}<1\right)$ is the inflection point of the curve $\boldsymbol{p}(t)$ if and only if the direction of $\boldsymbol{p}^{\prime}(t) \times \boldsymbol{p}^{\prime \prime}(t)$ changes when it passes through $t_{0}$. According to Equation (4), we have:

$$
\boldsymbol{p}^{\prime}(t) \times \boldsymbol{p}^{\prime \prime}(t)=f(t ; u, v)\left(\boldsymbol{q}_{1} \times \boldsymbol{q}_{3}\right)
$$

where:

$$
\begin{aligned}
f(t ; u, v) & =-\left|\begin{array}{cc}
B_{0}^{\prime}(t) & B_{3}^{\prime}(t) \\
B_{0}^{\prime \prime}(t) & B_{3}^{\prime \prime}(t)
\end{array}\right|+u\left|\begin{array}{cc}
B_{2}^{\prime}(t) & B_{3}^{\prime}(t) \\
B_{2}^{\prime \prime}(t) & B_{3}^{\prime \prime}(t)
\end{array}\right|+v\left|\begin{array}{cc}
B_{0}^{\prime}(t) & B_{1}^{\prime}(t) \\
B_{0}^{\prime \prime}(t) & B_{1}^{\prime \prime}(t)
\end{array}\right| \\
& =6 \lambda(2-\lambda) t^{2}+6 \lambda(\lambda-2) t+2 \lambda(3-\lambda)+6 \lambda t^{2} u+6 \lambda(t-1)^{2} v .
\end{aligned}
$$

As a result, $\boldsymbol{P}\left(t_{0}\right)\left(0<t_{0}<1\right)$ is an inflection point of the curve $\boldsymbol{p}(t)$ if and only if the sign of $f(t ; u, v)$ changes when it passes through $t_{0}$. In the $u v$-plane, the curve $\boldsymbol{p}(t)$ with the potential region of inflection points shall be covered with a family of straight lines. By the theory of envelopes [18], the envelope of the straight lines is:

$$
\left\{\begin{array}{l}
f(t ; u, v)=0 \\
f_{t}^{\prime}(t ; u, v)=0
\end{array}\right.
$$

That is:

$$
\left\{\begin{array}{l}
3 \lambda(2-\lambda) t^{2}+3 \lambda(\lambda-2) t+\lambda(3-\lambda)+3 \lambda t^{2} u+3 \lambda(t-1)^{2} v=0 \\
2 \lambda(2-\lambda) t+\lambda(\lambda-2)+2 \lambda t u+2 \lambda(t-1) v=0
\end{array}\right.
$$


It is not difficult to find that $u$ and $v$, given by Equation (6), are the solution to Equation(8), which means that the envelope of the straight lines is just the curve $C$.

As previously described, the curve $C$ is a strictly convex and continuous curve. Thus, the swept region of the tangent of the curve $C$ is $S \cup D \cup C$, that is, the potential region of inflection point(s). As shown in Figure 3, the region $D$ is composed of two asymptotes:

$$
u=\frac{\lambda}{3}-1, \quad v=\frac{\lambda}{3}-1
$$

and the curve $C$ (not including the curve $C$ ). The region $S$ includes two parts: one part is in the upper left part of the intersection of the two asymptotic lines, the other part is the lower right part of that. Given in Figure 3 are three different regional distributions of inflection point(s) corresponding to $\lambda=1$, $\lambda=2$, and $\lambda=3$.

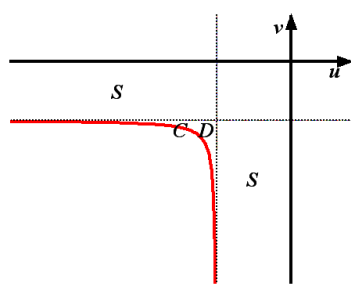

(a)

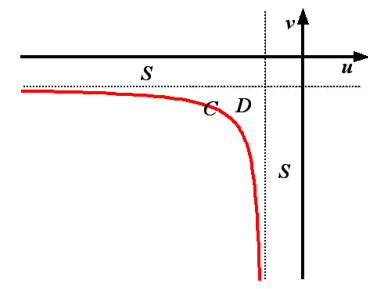

(b)

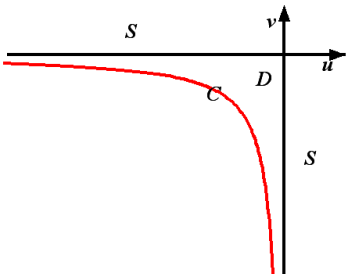

(c)

Figure 3. Regional distribution of inflection point $(S$, single inflection point region; $D$, double inflection points region); (a) $\lambda=1$; (b) $\lambda=2$; (c) $\lambda=3$.

For any point $\left(u_{0}, v_{0}\right) \in S \cup D \cup C$, at least one line $f\left(t_{0} ; u, v\right)=0$ passing through $\left(u_{0}, v_{0}\right)$ on the $u v$-plane is tangent to the curve $C$. Suppose $\left(u_{0}, v_{0}\right) \in C$ corresponds to the parameter $t_{0}$. Then we have $f\left(t_{0} ; u_{0}, v_{0}\right)=0$ and $f_{t}^{\prime}\left(t_{0} ; u_{0}, v_{0}\right)=0$. The Taylor expansion of $f\left(t ; u_{0}, v_{0}\right)$ about $t_{0}$ is:

$$
f\left(t ; u_{0}, v_{0}\right)=\frac{1}{2} f_{t t}^{\prime \prime}\left(t_{0} ; u_{0}, v_{0}\right)\left(t-t_{0}\right)^{2}+o\left(t-t_{0}\right)^{2}
$$

where:

$$
f_{t t}^{\prime \prime}\left(t_{0} ; u_{0}, v_{0}\right)=12 \lambda(2-\lambda)+12 \lambda u_{0}+12 \lambda v_{0}=\frac{2 \lambda^{2}}{t_{0}\left(t_{0}-1\right)} .
$$

For $\lambda \in(0,3], t_{0} \in(0,1)$, we get $f_{t t}^{\prime \prime}\left(t_{0} ; u_{0}, v_{0}\right) \neq 0$. Thus, the sign of $f\left(t ; u_{0}, v_{0}\right)$ does not change when it passes through $t_{0}$. There is no inflection point on the cubic parametric curve.

If $\left(u_{0}, v_{0}\right) \in S \cup D$, let $f\left(t_{0} ; u, v\right)=0$ be the straight line passing through $\left(u_{0}, v_{0}\right)$ and be tangent to the curve $C$. The Taylor expansion of $f\left(t ; u_{0}, v_{0}\right)$ about $t_{0}$ is:

$$
f\left(t ; u_{0}, v_{0}\right)=f_{t}^{\prime}\left(t_{0} ; u_{0}, v_{0}\right)\left(t-t_{0}\right)+o\left(t-t_{0}\right),
$$

where $f_{t}^{\prime}\left(t_{0} ; u_{0}, v_{0}\right) \neq 0$ (if $f_{t}^{\prime}\left(t_{0} ; u_{0}, v_{0}\right)=0$, then $\left(u_{0}, v_{0}\right) \in C$ ). As a result, the sign of $f\left(t ; u_{0}, v_{0}\right)$ changes when it passes through $t_{0}$. That is, $\boldsymbol{p}\left(t_{0}\right)$ is the inflection point of the curve $\boldsymbol{p}(t)$.

Furthermore, if $\left(u_{0}, v_{0}\right) \in S$, then there exists only one straight line that is tangent to the curve $C$ and passes through $\left(u_{0}, v_{0}\right)$, and the corresponding cubic parametric curve $p(t)$ has only one inflection point. If $\left(u_{0}, v_{0}\right) \in D$, then there exist two straight lines that are tangent to the curve $C$ and pass through $\left(u_{0}, v_{0}\right)$, and the corresponding curve $\boldsymbol{p}(t)$ has double inflection points.

Figure 3 shows that double inflection region of the Ball curve is smaller than that of the cubic Bézier curve. However, single inflection regions of these two kinds of curves are of the same size. 


\subsubsection{About the Double Point}

The curve $\boldsymbol{p}(t)$ has a double point if and only if there are $0 \leq t_{1}<t_{2} \leq 1$ such that:

$$
\boldsymbol{p}\left(t_{1}\right)-\boldsymbol{p}\left(t_{2}\right)=0,
$$

which, according to Equation (4), leads to the following system of equations:

$$
\left\{\begin{array}{l}
u=\frac{B_{0}\left(t_{2}\right)-B_{0}\left(t_{1}\right)}{B_{2}\left(t_{2}\right)+B_{3}\left(t_{2}\right)-B_{2}\left(t_{1}\right)-B_{3}\left(t_{1}\right)}, \\
v=\frac{B_{3}\left(t_{1}\right)-B_{3}\left(t_{2}\right)}{B_{2}\left(t_{2}\right)+B_{3}\left(t_{2}\right)-B_{2}\left(t_{1}\right)-B_{3}\left(t_{1}\right)},
\end{array}\right.
$$

where $\left(t_{1}, t_{2}\right) \in \Delta=\left\{\left(t_{1}, t_{2}\right) \in R^{2} \mid 0 \leq t_{1}<t_{2} \leq 1\right\}$.

The system of Equation (9) defines a topological mapping $F: \Delta \subset \boldsymbol{R}^{2} \rightarrow F(\Delta) \subset \boldsymbol{R}^{2}$. The image region $L=F(\Delta)$ is a simply connection region in the $u v$-plane. The three boundary lines of the region $\Delta: t_{1}=t_{2}, t_{1}=0$, and $t_{2}=1$ correspond to the three boundary curves of the image region $L$ : the curve $C$ (does not belong to $L$ ), the curve $L_{1}$ and $L_{2}$ (both belong to $L$ ), where:

$$
\begin{aligned}
& L_{1}:\left\{\begin{array}{l}
u=\frac{\lambda(t-1)^{2}}{t(2 t-3)}-1, \quad(0<t \leq 1), \\
v=\frac{\lambda(t-1)}{2 t-3}-1,
\end{array}\right. \\
& L_{2}:\left\{\begin{array}{rl}
u & =\frac{\lambda t}{2 t+1}-1, \\
v & =\frac{\lambda t^{2}}{(t-1)(2 t+1)}-1,
\end{array} \quad(0 \leq t<1) .\right.
\end{aligned}
$$

For the curve $L_{1}, \lambda \in(0,3], 0<t<1$, we know that:

$$
\frac{\mathrm{d} v}{\mathrm{~d} u}=-\frac{t^{2}}{(t-1)(t-3)}<0, \frac{\mathrm{d}^{2} v}{\mathrm{~d} u^{2}}=\frac{2}{\lambda} \cdot\left[\frac{t(2 t-3)}{(t-1)(t-3)}\right]^{3}<0, \lim _{t \rightarrow 0^{+}} u=-\infty \lim _{t \rightarrow 0^{+}} v=\frac{\lambda}{3}-1
$$

For the curve $L_{2}$, similarly:

$$
\frac{\mathrm{d} v}{\mathrm{~d} u}=-\frac{t(t+2)}{(t-1)^{2}}<0, \frac{\mathrm{d}^{2} v}{\mathrm{~d} u^{2}}=\frac{2}{\lambda} \cdot\left(\frac{2 t+1}{t-1}\right)^{3}<0, \lim _{t \rightarrow 1^{-}} u=\frac{\lambda}{3}-1, \lim _{t \rightarrow 1^{-}} v=-\infty .
$$

As a result, both the curves $L_{1}$ and $L_{2}$ are monotonically decreasing and strictly convex continuous curves. The curve $L_{1}$ intersects the curve $L_{2}$ at the point $(-1,-1)$. Additionally, the curve $L_{1}$ has the asymptote $v=\frac{\lambda}{3}-1$ and the curve $L_{2}$ has the asymptote $u=\frac{\lambda}{3}-1$, and the curve $C$ does not intersect $L_{1}$ and $L_{2}$, as shown in Figure 4.

In summary, the curve $C$ (does not belong to $L$ ), and the curves $L_{1}$ and $L_{2}$ (both belong to $L$ ) round into the simply connected region $L$. If $\left(u_{0}, v_{0}\right) \in L$, the corresponding cubic parametric curve $p(t)$ has only one double point.

Example 1. For a given set of control points $\boldsymbol{P}_{i} \in \boldsymbol{R}^{2}(i=0,1,2,3)$, a few different cubic parametric curves contain singularities that can be designed according to the conditions discussed above. Figure 4 illustrates that the singularity can be removed by changing the value of the shape factor $\lambda$.

Figure 4a shows two segment cubic curves containing double inflection points when $\lambda=2.4$ and $\lambda=3$. Figure $4 \mathrm{~b}$ is two segment cubic curves containing a single inflection point. Figure $4 \mathrm{c}$ is two segment cubic curves containing a double point. For the same control polygon, Figure $4 \mathrm{~d}-\mathrm{f}$ denote cubic curves containing double inflection points, cusp, double point, respectively. In particular, when $\lambda=3$, the red curve is a cubic Bézier curve in Figure 4. 


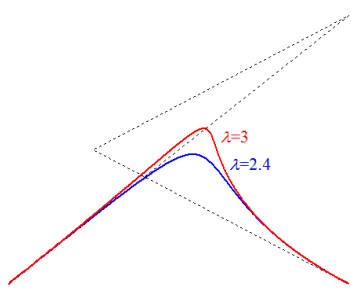

(a)

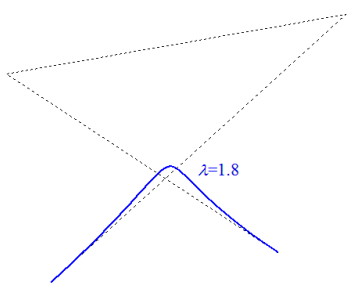

(d)

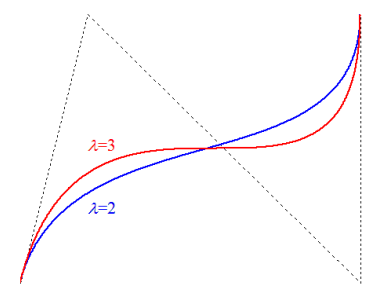

(b)

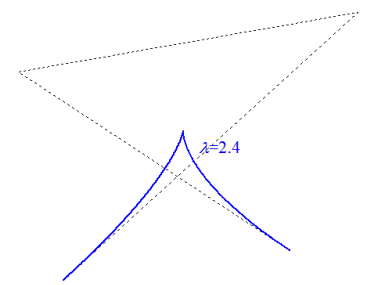

(e)

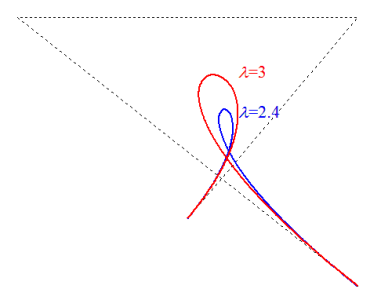

(c)

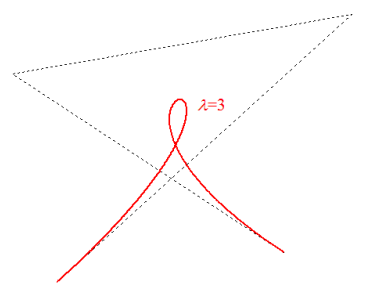

(f)

Figure 4. The position of the singularity is adjusted by changing the shape factor; (a) Double inflection points; (b) Single inflection point; (c) Double point; (d) Double inflection points; (e) Cusp; (f) Double point.

Figure 4 tells us that we can construct the curve with the desired geometric characteristics by adjusting the shape factor value. The cubic parametric curve can construct more abundant geometric characteristics than the cubic Bézier curve in geometric design.

\subsubsection{About the Convexity}

We will discuss the case of $(u, v) \in N=R^{2} \backslash(C \cup S \cup D \cup L)$, where is no cusp, double point, or inflection point on the cubic parameter curve, and the direction of the binormal vector $\boldsymbol{p}^{\prime}(t) \times \boldsymbol{p}^{\prime \prime}(t)$ does not change.

The upper left part of the area surrounded by the curves $L_{1}$ and $L_{2}$ (not including the curves $L_{1}$ or $L_{2}$ ) is marked as $N_{1}$, and the lower right part of the area surrounded by the curves $L_{1}$ and $L_{2}$ is marked as $N_{2}$. Set $N_{0}=N \backslash\left(N_{1} \cup N_{2}\right)$, as shown in Figure 4 .

Let:

$$
\begin{gathered}
\boldsymbol{m}(t)=\boldsymbol{p}^{\prime}(0) \times[\boldsymbol{p}(t)-\boldsymbol{p}(0)]=\varphi(t ; u, v)\left(\boldsymbol{q}_{1} \times \boldsymbol{q}_{3}\right), \\
\boldsymbol{n}(t)=[\boldsymbol{p}(t)-\boldsymbol{p}(0)] \times \boldsymbol{p}^{\prime}(t)=\psi(t ; u, v)\left(\boldsymbol{q}_{1} \times \boldsymbol{q}_{3}\right) .
\end{gathered}
$$

According to Equations (4) and (5), we have:

$$
\begin{gathered}
\varphi(t ; u, v)=\lambda\left\{B_{3}(t)+v\left[B_{2}(t)+B_{3}(t)\right]\right\}=\lambda t^{2}[(3-\lambda)+(\lambda-2) t+(3-2 t) v] . \\
\psi(t ; u, v)=\left[1-B_{0}(t)\right] B_{3}^{\prime}(t)+B_{3}(t) B_{0}^{\prime}(t)+u\left\{\left[B_{2}(t)+B_{3}(t)\right] B_{3}^{\prime}(t)-\left[B_{2}^{\prime}(t)+B_{3}^{\prime}(t)\right] B_{3}(t)\right\} \\
+v\left\{\left[1-B_{0}(t)\right]\left[B_{2}^{\prime}(t)+B_{3}^{\prime}(t)\right]+B_{0}^{\prime}(t)\left[B_{2}(t)+B_{3}(t)\right]\right\} .
\end{gathered}
$$

For any $t_{0} \in(0,1)$, if none of the directions of the vectors $\boldsymbol{m}(t), \boldsymbol{n}(t)$, and $\boldsymbol{p}^{\prime}(t) \times \boldsymbol{p}^{\prime \prime}(t)$ changes when they pass through $t_{0}$, the curve $\boldsymbol{p}(t)$ is globally convex. If the direction of the binormal vector $\boldsymbol{p}^{\prime}(t) \times \boldsymbol{p}^{\prime \prime}(t)$ does not change when it passes through $t_{0}$, but the direction of $\boldsymbol{m}(t)$ or $\boldsymbol{n}(t)$ changes, then the curve $p(t)$ is locally convex [13].

As described above, if $(u, v) \in N=N_{0} \cup N_{1} \cup N_{2}$, the sign of the function $f(t ; u, v)$ does not change, and the direction of the binormal vector $\boldsymbol{p}^{\prime}(t) \times \boldsymbol{p}^{\prime \prime}(t)$ does not change.

From Equation (12), if: 


$$
v=-\frac{B_{3}\left(t_{0}\right)}{B_{2}\left(t_{0}\right)+B_{3}\left(t_{0}\right)}=\frac{\lambda}{2}-1+\frac{\lambda}{2\left(2 t_{0}-3\right)},
$$

Then $\varphi\left(t_{0} ; u, v\right)=0$, and the direction of the vector $m(t)$ changes when it passes through $t_{0}$ and the range of $v$ is $-1<v<\frac{\lambda}{3}-1$. Thus, if $(u, v) \in N_{1}$, the direction of either $\boldsymbol{p}^{\prime}(t) \times \boldsymbol{p}^{\prime \prime}(t)$, or $\boldsymbol{n}(t)$ does not change when they pass through $t_{0}$, but the direction of $\boldsymbol{m}(t)$ changes, the curve $\boldsymbol{p}(t)$ is locally convex. In fact, $N_{1}$ happens to be the area covered by the tangent of $L_{2}$ in the region $N$.

Similarly, solving the equations:

$$
\left\{\begin{array}{c}
\psi(t ; u, v)=0 \\
\psi_{t}^{\prime}(t ; u, v)=0
\end{array}\right.
$$

for $u, v$ verifies that the envelope of the family of straight lines $\psi(t ; u, v)=0$ happens to be the curve $L_{1}$. If $(u, v) \in N_{2}$, the direction of either $\boldsymbol{p}^{\prime}(t) \times \boldsymbol{p}^{\prime \prime}(t)$ or $\boldsymbol{m}(t)$ does not change when they pass through $t_{0}$, but the direction of $\boldsymbol{n}(t)$ changes, so the curve $\boldsymbol{p}(t)$ is locally convex. The region $N_{2}$ is the area covered by the tangent of $L_{1}$ in the region $N$. As shown in Figure 5, where $l_{1}: v=-1(u<-1)$ and $l_{2}: u=-1(v<-1)$.

If $(u, v) \in N_{0}$, none of the directions of $\boldsymbol{m}(t), \boldsymbol{n}(t)$, and $\boldsymbol{p}^{\prime}(t) \times \boldsymbol{p}^{\prime \prime}(t)$ changes when they pass through $t_{0}$. Therefore, the curve $p(t)$ is globally convex.

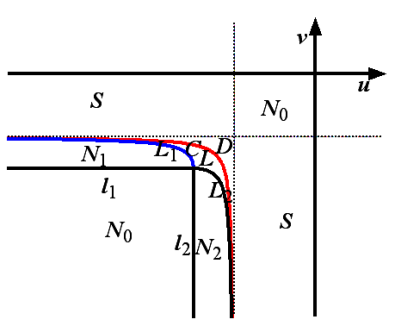

(a) $\lambda=1$

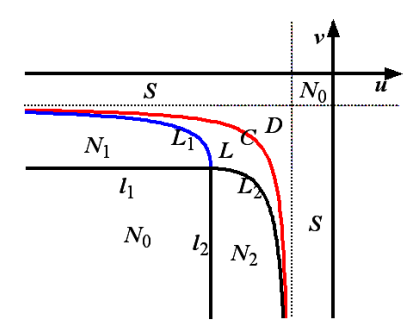

(b) $\lambda=2$

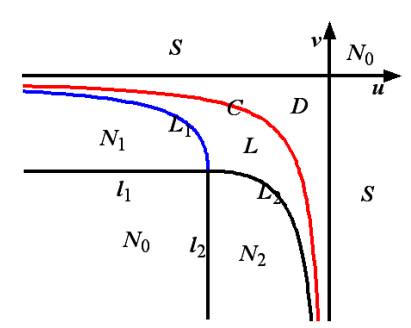

(c) $\lambda=3$

Figure 5. The shape distribution of the cubic parameter curve $(C$ is the cusp region; $L$ is the double point region; $S$ is the single inflection point region; $D$ is the double inflection points region; $N_{0}$ is the global convexity region; $N_{1} \cup N_{2}$ is the local convexity region). (a) $\lambda=1$; (b) $\lambda=2$; (c) $\lambda=3$.

In summary, we have the following conclusions:

Theorem 2. When edge vectors $\boldsymbol{q}_{1}$ and $\boldsymbol{q}_{3}$ are non-parallel, let $\boldsymbol{q}_{2}=u \boldsymbol{q}_{1}+v \boldsymbol{q}_{3}$. Shape features of the plane cubic parametric curve $p(t)$ depend on the following distribution of points $(u, v)$ in the uv-plane (as shown in Table 1).

Table 1. The shape distribution of the plane cubic parameter curve.

\begin{tabular}{ccccc}
\hline \multirow{2}{*}{$(\boldsymbol{u}, \boldsymbol{v})$} & \multicolumn{4}{c}{ Shape Features of the Plane Cubic Parametric Curve $\boldsymbol{p}(\boldsymbol{t})$} \\
\cline { 2 - 5 } & Convexity & Cusp & Double Point & Inflection Point \\
\hline$C$ & $/$ & one & no & no \\
$L$ & $/$ & no & one & no \\
$S$ & $/$ & no & no & one \\
$D$ & $/$ & no & no & two \\
$N_{0}$ & global convexity & no & no & no \\
$N_{1} \cup N_{2}$ & local convexity & no & no & no \\
\hline
\end{tabular}




\subsection{Edge Vectors $\boldsymbol{q}_{1}$ and $\boldsymbol{q}_{3}$ Are Parallel}

If $\boldsymbol{q}_{1} \| \boldsymbol{q}_{3}$, without loss of generality, edge vectors $\boldsymbol{q}_{1}$ and $\boldsymbol{q}_{2}$ are the base vectors of the plane. Let $\boldsymbol{q}_{3}=\alpha \boldsymbol{q}_{1}$. From Equation (3), we have:

$$
\boldsymbol{p}(t)=\boldsymbol{P}_{0}+\left[1-B_{0}(t)+\alpha B_{3}(t)\right] \boldsymbol{q}_{1}+\left[B_{2}(t)+B_{3}(t)\right] \boldsymbol{q}_{2} .
$$

\subsubsection{About the Cusp}

We discuss the curve $\boldsymbol{p}(t)$ similarly to Section 4.1.1 The curve $\boldsymbol{p}(t)$, having a cusp, is equivalent to $p^{\prime}(t)=0, t \in(0,1)$. From Equation (14), we have:

$$
\boldsymbol{p}^{\prime}(t)=\left[-B_{0}^{\prime}(t)+\alpha B_{3}^{\prime}(t)\right] \boldsymbol{q}_{1}+\left[B_{2}^{\prime}(t)+B_{3}^{\prime}(t)\right] \boldsymbol{q}_{2} .
$$

Since edge vectors $q_{1}$ and $q_{2}$ are linearly independent, we know $p^{\prime}(t)=0, t \in(0,1)$ is equivalent to:

$$
\left\{\begin{aligned}
{\left[3(\lambda-2) t^{2}+2(3-2 \lambda) t+\lambda\right]+\alpha\left[3(\lambda-2) t^{2}+2(3-\lambda) t\right] } & =0 \\
6 t(1-t) & =0
\end{aligned}\right.
$$

It is obvious that Equation (15) has no solution for $t$ in $(0,1)$. Thus, the plane cubic parameter curve $p(t)$ has no cusp.

\subsubsection{About the Inflection Point}

The point $\boldsymbol{p}\left(t_{0}\right)\left(0<t_{0}<1\right)$ is the inflection point of the curve $\boldsymbol{p}(t)$ if, and only if, the direction of $p^{\prime}(t) \times p^{\prime \prime}(t)$ changes when it passes through $t_{0}$. According to Equation (14), we have:

$$
\boldsymbol{p}^{\prime}(t) \times \boldsymbol{p}^{\prime \prime}(t)=f(t ; \alpha)\left(\boldsymbol{q}_{1} \times \boldsymbol{q}_{2}\right),
$$

where:

$$
\begin{aligned}
f(t ; \alpha) & =-\left|\begin{array}{rr}
B_{0}^{\prime}(t) & B_{2}^{\prime}(t)+B_{3}^{\prime}(t) \\
B_{0}^{\prime \prime}(t) & B_{2}^{\prime \prime}(t)+B_{3}^{\prime \prime}(t)
\end{array}\right|+\alpha\left|\begin{array}{rr}
B_{3}^{\prime}(t) & B_{2}^{\prime}(t)+B_{3}^{\prime}(t) \\
B_{3}^{\prime \prime}(t) & B_{2}^{\prime \prime}(t)+B_{3}^{\prime \prime}(t)
\end{array}\right| \\
& =6 \lambda\left[(1-t)^{2}-\alpha t^{2}\right] .
\end{aligned}
$$

When $\alpha>0$ :

$$
f_{t}^{\prime}(t ; \alpha)=12 \lambda(t-1-\alpha t)<0 .
$$

since:

$$
f(0 ; \alpha)=6 \lambda>0
$$

and:

$$
f(1 ; \alpha)=-6 \lambda \alpha<0,
$$

the sign of $f(t ; \alpha)$ changes when it passes through a unique $t_{0}=\frac{1}{\sqrt{\alpha}+1}$. If and only if $\alpha>0$ (i.e., the direction of $\boldsymbol{q}_{1}$ is the same as that of $\boldsymbol{q}_{3}$ ), the cubic parametric curve $\boldsymbol{p}(t)$ has one, and only one, inflection point, otherwise the four control points are collinear.

\subsubsection{About the Double Point}

The curve $\boldsymbol{p}(t)$ has double points if, and only if, $0 \leq t_{1}<t_{2} \leq 1$ such that $\boldsymbol{p}\left(t_{1}\right)-\boldsymbol{p}\left(t_{2}\right)=0$, which leads to the following system of equations by Equation (14):

$$
\left\{\begin{array}{l}
\alpha=\frac{B_{0}\left(t_{2}\right)-B_{0}\left(t_{1}\right)}{B_{3}\left(t_{2}\right)-B_{3}\left(t_{1}\right)} \\
B_{2}\left(t_{2}\right)+B_{3}\left(t_{2}\right)=B_{2}\left(t_{1}\right)+B_{3}\left(t_{1}\right),
\end{array}\right.
$$


where the second equation can be written as:

$$
t_{1}^{2}\left(3-2 t_{1}\right)=t_{2}^{2}\left(3-2 t_{2}\right)
$$

Equation (16) implies that there exists $\eta \in\left(t_{1}, t_{2}\right) \subseteq[0,1]$ such that $\left.\left(3 t^{2}-2 t^{3}\right)^{\prime}\right|_{t=\eta}=0$, i.e., $\eta(1-\eta)=0$, which contradicts $\eta(1-\eta)>0$. Hence, the plane cubic parametric curve $\boldsymbol{p}(t)$ has no double point.

To sum up, we have the following conclusions:

Theorem 3. Suppose $q^{1}|| q^{3}$.

(1) The cubic parametric curve of $\boldsymbol{p}(t)$ has no cusp or double point.

(2) If, and only if, when $\alpha>0$ (i.e., the direction of $\boldsymbol{q}_{1}$ is the same as that of $\boldsymbol{q}_{3}$ ), the cubic parametric curve $p(t)$ has one and only one, inflection point, otherwise the four control points are collinear.

\section{The Influence of the Shape Factor on the Cubic Parametric Curve}

According to Theorems 2 and 3, We can further discuss the influence of the shape factor $\lambda$ on the cubic curve $p(t)$. The change of the shape factor affects almost all regions. For example, when the curve $p(t)$ has only one inflection point, we can adjust the shape factor $\lambda$ to eliminate it. Thus, by adjusting the shape factor $\lambda$, one can control the shape of the curve flexibly, which brings about significant convenience in practical geometric design.

(1) Shape distribution of the cubic parametric curve $p(t)$ is symmetric about the straight line $u=v$.

(2) When $\lambda=0$, the cubic parametric curve $p(t)$ reduces to a straight line, and the effect of shape factor $\lambda$ disappears. When $\lambda=2, p(t)$ degenerates into the Ball curve. If $\lambda=3, \boldsymbol{p}(t)$ degenerates into the cubic Bézier curve.

(3) As the shape factor $\lambda$ increases, the curve $C$ is drawn towards the origin $(0,0)$, the curve $L_{1}$ is pulled toward the u-axis, and $L_{2}$ is pulled toward the v-axis. Thus, the region $S$ and $N_{0}$ decrease, and the regions $D, N_{1} \cup N_{2}$, and $L$ increase gradually.

(4) When

$$
(u, v) \in\{(u, v) \mid-1 \leq u, v<0\} \backslash\{(-1,-1)\},
$$

then the first edge and the last edge of the control polygon intersects (except that the first point and the last point coincide), there are likely singularity points, single inflection points, or double inflection points on the curve $\boldsymbol{p}(t)$. Additionally, the curve $\boldsymbol{p}(t)$ may also be globally convex, but cannot be locally convex. Adjusting the shape factor can make the curve $p(t)$ become a global convex curve.

\section{Conclusions}

In this paper, we construct a class of cubic parametric curves with a variable shape factor. The Ball curve, cubic Bézier curve, and cubic Timmer curve are special cases of the curve. Geometric features of this cubic parametric curve with a shape factor are analyzed by means of the theory of envelope and topological mapping. The effects of the shape factor on the cubic parametric curve are made clear. Necessary and sufficient conditions are derived for this curve to have one or two inflection points, a loop or a cusp, or to be locally or globally convex. Those conditions are completely characterized by the relative position of the edge vectors of the control polygon and the shape factor. The results are summarized in a shape diagram. The conditions are useful for classifying and modifying the cubic parametric curve. 
Acknowledgments: The authors would like to thank the referees for their valuable comments which greatly help improve the clarity and quality of the paper. This work was supported in part by the National Natural Science Foundation of China (Grant No. 61472466 and 11471093), Key Project of Scientific Research, Education Department of Anhui Province of China under Grant No. KJ2014ZD30. The Fundamental Research Funds for the Central Universities under Grant No. JZ2015HGXJ0175.

Author Contributions: Zhi Liu conceived and designed the experiments; Xiaoyan Chen performed the experiments; Jieqing Tananalyzed the data; Chen Li wrote the paper.

Conflicts of Interest: The authors declare no conflict of interest.

\section{References}

1. Goldman, R.; Simeonov, P. Two essential properties of (q, h)-Bernstein-Bézier curves. Appl. Numer. Math. 2015, 96, 82-93. [CrossRef]

2. Bézier, P. UNISURE, from styling to tool-shop. Comput. Ind. 1983, 4, 115-126. [CrossRef]

3. Ball, A.A. CONSURF Part 1: Introduction of the Conic Lofting Title. Comput.-Aided Des. 1974, 6, $243-349$. [CrossRef]

4. Ball, A.A. CONSURF Part 2: Description of the Algorithms. Comput.-Aided Des. 1975, 7, 237-242. [CrossRef]

5. Ball, A.A. CONSURF Part 3: How the program is used. Comput.-Aided Des. 1977, 9, 9-12. [CrossRef]

6. Said, H.B. A generalized Ball curve and its recursive algorithm. ACM Trans. Graph. 1989, 4, 360-378. [CrossRef]

7. Savetseranee, D.; Dejdumrong, N. Monomial forms of two generalized ball curves and their proofs. In Proceedings of the 2013 10th International Joint Conference on Computer Science and Software Engineering (JCSSE), KhonKaen, Thailand, 29-31 May 2013; pp. 235-239.

8. Goodman, T.N.T.; Said, H.B. Properties of two types of generalized Ball curves and surfaces. Comput.-Aided Des. 1991, 23, 554-560. [CrossRef]

9. Goodman, T.N.T.; Said, H.B. Shape-preserving properties of the generalized Ball basis. Comput.-Aided Des. 1991, 8, 115-121. [CrossRef]

10. Kim, D.S. Hodograph approach to geometric characterization of parametric cubic curves. Comput.-Aided Des. 1993, 25, 644-654. [CrossRef]

11. Li, Y.M.; Cripps, R.J. Identification of inflection points and cusps on rational curves. Comput.-Aided Des. 1997, 14, 491-497. [CrossRef]

12. Manocha, D.; Canny, J.F. Detecting cusps and inflection points in curves. Comput. Aided Geom. Des. 1992, 9, 1-24. [CrossRef]

13. Yang, Q.; Wang, G. Inflection points and singularities on C-curves. Comput. Aided Geom. Des. 2004, 21, 207-213. [CrossRef]

14. Juhász, I. On the singularity of a class of parametric curves. Comput. Aided Geom. Des. 2006, 23, 146-156. [CrossRef]

15. Stone, M.C.; DeRose, T.D. A geometric characterization of parametric cubic curves. ACM Trans. Graph. 1989, 8, 147-163. [CrossRef]

16. OruÇ, H.; Phillips, G.M. q-Bernstein polynomials and Bézier curves. J. Comput. Appl. Math. 2003, 151, 1-12. [CrossRef]

17. Timmer, H.G. Alternative representation for parametric cubic curves and surfaces. Comput.-Aided Des. 1980, 12, 25-28. [CrossRef]

18. Han, X.A.; Huang, X.L.; Ma, Y.C. Shape analysis of cubic trigonometric Bézier curves with a shape parameter. Appl. Math. Comput. 2010, 217, 2527-2533. [CrossRef]

(C) 2017 by the authors; licensee MDPI, Basel, Switzerland. This article is an open access article distributed under the terms and conditions of the Creative Commons Attribution (CC BY) license (http:/ / creativecommons.org/licenses/by/4.0/). 